\title{
Observation of a New Type of THz Resonance of Surface Plasmons Propagating on Metal-Film Hole Arrays
}

\author{
Dongxia Qu and D. Grischkowsky \\ School of Electrical and Computer Engineering, Oklahoma State University, Stillwater, Oklahoma 74078, USA
}

(Received 9 April 2004; published 4 November 2004)

\begin{abstract}
Highly conducting metal-film subwavelength hole arrays, lithographically fabricated on highresistivity silicon wafers in optical contact with thick silicon plates, have been characterized by terahertz time-domain spectroscopy with subpicosecond resolution and over a frequency range from 0.5 to $3 \mathrm{THz}$ with $5 \mathrm{GHz}$ resolution. A well-defined ringing structure extending to more than $250 \mathrm{psec}$ is observed on the trailing edge of the transmitted $\mathrm{THz}$ pulse. In the frequency domain this ringing structure corresponds to a new type of extremely sharp resonant line structure between the fundamental surface plasmon modes of the hole array. A simple theoretical model is presented and shows good agreement with the experimental data.
\end{abstract}

DOI: 10.1103/PhysRevLett.93.196804

Although the microwave and terahertz transmission through thin-metal films or metal foils perforated periodically with apertures has been studied since 1967 [16], unusual high-transmission resonances through subwavelength hole arrays were only recently discovered $[7,8]$ at optical frequencies. These resonances occurred at wavelengths different from those predicted by the classic diffraction theory [2]. Since this discovery much work has been done to experimentally characterize the transmission of thin subwavelength hole arrays in the optical [9-21], infrared [22], and $\mathrm{THz}$ [23-28] regions. The enhanced transmission is now understood to be due to the excitation of surface plasmon (SP) resonances [7$22,24,25,27-33]$. The SP enhancement can be affected by the refractive index of the adjacent medium $[9,12]$, the shape and orientation of the holes [10,20,27], and the thickness of the metal film [16]. In all these measurements, SP enhanced transmission has been observed only at the frequencies determined by the integral order SP modes coupled to the array [7,8]. Here, we present observations of a new type of high-transmission, extremely narrow resonances at frequencies between those of the integral order SP modes. These resonances correspond to SP propagation in directions different than those allowed by the integral order modes. We call these resonances fractional order surface plasmons (FSP), which are made possible by the high conductivity of metal films at $\mathrm{THz}$ frequencies.

In this Letter, we present a time-domain and frequency-domain study of the amplitude and phase transmission properties of a thin-metal-film subwavelength hole array, lithographically fabricated on a silicon wafer. The utilized $\mathrm{THz}$ time-domain spectroscopy (THz-TDS) technique measures the complete THz electric field pulse with subpicosecond time resolution [34]. For this characterization a subpicosecond $\mathrm{THz}$ pulse is incident on the hole array. The transmitted $\mathrm{THz}$ pulse consisted of an attenuated subpicosecond pulse due to the
PACS numbers: 73.20.Mf, 41.20.Jb, 42.25.Fx, 78.47.+p

classical electromagnetic transmission of the array, followed by a dramatic ringing structure, extending to approximately $250 \mathrm{psec}$ and due to the SPs excited by the incident pulse. The observed ringing provides a measure of the time dependence and lifetime of the generated, frequency-dependent, standing waves spatially coherent with the array and composed of counterpropagating, SPs.

The complex numerical Fourier transforms of the measured $\mathrm{THz}$ pulses give both spectral amplitude and phase. The long temporal scans of the pulse measurement yields a frequency resolution of $5 \mathrm{GHz}$. We observe a complex spectral structure for the output pulses compared to the smooth featureless spectrum of the input pulses. The transmission of the array is strongly enhanced by the lowest order SP with a peak power transmission 4 times greater than the geometrical transmission. In the frequency domain, the extensive ringing on the trailing edge of the transmitted pulses corresponds to amplitude and phase resonances in the region between the lowest order SP modes of the hole array.

In the THz-TDS measurement, the input $\mathrm{THz}$ pulse, polarized along the $x$ axis, is incident on the metal face of the sample at the beam waist. To a good approximation, the incoming $\mathrm{THz}$ beam has a linearly wavelengthdependent Gaussian profile with an $9.2 \mathrm{~mm}$ diameter beam waist (1/e point in amplitude) at $1 \mathrm{THz}$. The metal hole array is a lithographically fabricated $280-\mathrm{nm}$-thick Al layer deposited onto a doubleside polished $0.32-\mathrm{mm}$ thick, high-resistivity $\mathrm{Si}$ wafer. The $60 \mu \mathrm{m}$ period of the hole array has submicron precision with the rectangular hole dimensions of $15 \mu \mathrm{m}(x$ axis $) \times 30 \mu \mathrm{m}$ ( $y$ axis $)$. The ratio of hole area to the total area is $1 / 8$, equal to the geometric power transmission. A 10 -mm-thick highresistivity Si plate was placed in optical contact with the backside of the wafer to eliminate the backside reflection, thereby enabling clean observations of plasmon dynamics to an unprecedented $250 \mathrm{psec}$. 
The sample is centered with a $30-\mathrm{mm}$-diameter aperture. The empty aperture is used to obtain the reference $\mathrm{THz}$ pulse shown in Fig. 1(a). The subpicosecond input reference pulse is much shorter than the damping time of the SP resonances at metal-film hole array interface, which are responsible for the ringing on the transmitted THz sample pulse also shown in Fig. 1(a). The inset shows the temporal oscillations in more detail. Because the expected attenuation lengths of the SP waves are more than $100 \mathrm{~cm}$ [27], the decay of the ringing is considered to be mainly due to the initially overlapping FSP counter-
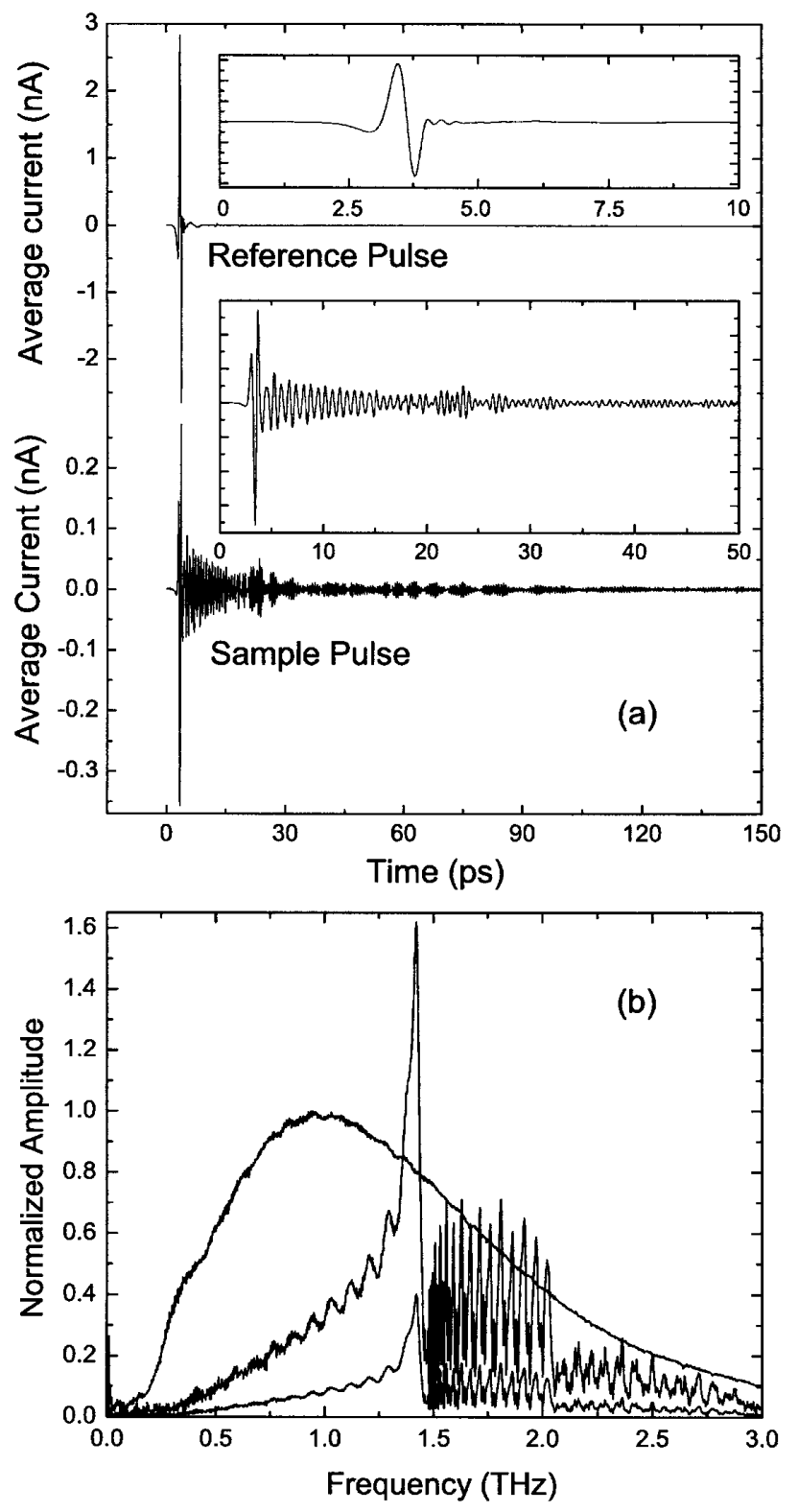

FIG. 1. (a) The measured reference pulse and the transmitted sample pulse. The insets show the expanded reference pulse between $0-10 \mathrm{ps}$ and the sample pulse between $0-50 \mathrm{ps}$. (b) The normalized amplitude spectra of the reference and sample pulses. propagating waves separating from each other due to the finite frequency-dependent excitation beam diameters. This separation causes the resulting FSP standing waves to disappear.

The corresponding amplitude spectra of the reference and sample pulses are shown in Fig. 1(b). These numerical Fourier transforms have approximately $5 \mathrm{GHz}$ resolution due to the total scan lengths of $223 \mathrm{psec}$, which include no backside reflection. The reference spectrum has been normalized to unity, and with respect to this same normalization the signal spectrum is shown as the lower curve. This signal spectrum, multiplied by the factor $1 /(0.700 \times 0.354)$ to remove the effects of the amplitude transmission of the silicon wafer-plate combination and the array, is shown as the upper curve.

The experimental transmission and phase change were calculated by taking the ratio and phase difference between the complex amplitude spectra of the sample and reference pulses as shown in Fig. 2, using the same normalization as for the upper sample spectrum of Fig. 1(b). Strongly enhanced transmission is seen at the first integral SP mode at $1.46 \mathrm{THz}$. The amplitude enhancement value of 2 , is equivalent to a power enhancement of 4 compared to the geometrical transmission.

In addition to the peaks predicted as the integral SP modes indicated by the three vertical dashed lines in the Fig. 2, we observed a series of strong and sharp transmission and phase change resonances in the frequency range between the 1.46 and $2.06 \mathrm{THz}$ resonances of the SP metal-silicon $[ \pm 1,0]$ and $[ \pm 1, \pm 1]$ modes, respectively. These features illustrated on an expanded scale in Figs. 2(b) and 2(d), will now be shown to be due to the coupling of the FSP standing waves to the hole array.

For a two-dimensional $x-y$ hole array illuminated by an impinging wave propagating in the $z$ direction, the SP coupling condition is given by $k_{s p}= \pm m k_{x} \pm n k_{y}$, where $k_{x}$ and $k_{y}$ are the array momentum wave vectors with $\left|k_{x}\right|=\left|k_{y}\right|=2 \pi / L$ ( $L$ is the period of the array), and $m$, $n$ are integers, representing the $m$ and $n$ diffracted orders from the hole array [7]. This relationship shows that for a given SP wave vector, resonant coupling occurs when the SP wave vector matches the in-plane momentum wave vectors provided by the hole array. In the $\mathrm{THz}$ domain, for a smooth highly conducting metal film the relation between the excited SP wave vector $k_{s p}$ and the free space vector $k_{0}$ is $k_{s p} \approx k_{0} \sqrt{\varepsilon}$ [27], where $\varepsilon$ is the dielectric constant of the media.

An intuitive understanding of the coupling between the SP and the illuminated array is based on the simple picture that the electromagnetic fields in the holes of the array excite SP standing waves consisting of two counterpropagating SP waves. The rectangular holes of the array are oriented along the $x$ and $y$ axes with the narrow dimension along the $x$ axis, thereby preserving the incident $x$ polarization. The FSP propagation vector is written 
in the general form $\mathbf{k}_{f s p}=k_{f s p}[\mathbf{x} \cos \theta+\mathbf{y} \sin \theta]$, where $\theta$ is the angle between the FSP propagation direction and the $x$ axis. We take the $\mathbf{x}$ component of the propagation vector to be $k_{f s p} \cos \theta= \pm 2 \pi / L$, which assumes $m=$ \pm 1 for the lowest order SP propagation in the $x$ direction. For a given $\theta$ the magnitude of $k_{f s p}$ is determined by this relationship. The measured transmission peaks shown in the frequency domain in Fig. 2(b) can be converted to angle by the above relationship and understood as the variation of the coupling of the FSP standing wave to the hole array as a function of the propagation direction $\theta$. For a given $\theta$, the corresponding free-space wavelength is $\lambda_{\text {Res }}=L n_{2} \cos \theta$.

For evaluation of the coupling we assume that two counter propagating FSPs interfere with each other and form a pure cosine FSP standing wave in the direction $\theta$ with the corresponding resonance frequency $c / \lambda_{\text {Res }}$. The normalized FSP standing-wave electric field $E_{\|}^{f s p}$ can be expressed as

$$
E_{\|}^{f s p}=\cos \left[k_{f s p} \cos \theta\left(x-x_{0}\right)+k_{f s p} \sin \theta\left(y-y_{0}\right)\right],
$$

where $x_{0}$ and $y_{0}$ represent the system self-determined relative position of the standing-wave nodes on the hole pattern. For our simulation we obtained the best fit with $x_{0}=-22 \mu \mathrm{m}$ and $y_{0}=-11.5 \mu \mathrm{m}$, which is quite close to the center between four neighboring holes $(-22.5 \mu \mathrm{m},-15 \mu \mathrm{m})$. The origin of coordinates is at the corner of the rectangular hole defined by the corner coordinates in microns $(0,0),(0,30),(15,0)$, and $(15,30)$.

The well-defined FSP standing waves are coupled to the $\mathrm{THz}$ wave transmitted through the periodic metallic hole array. The measured amplitude transmission is determined by the coupling efficiency $\operatorname{CE}(\theta)$ between the FSPs standing wave and the hole pattern of the array, as a function of $\theta \cdot \operatorname{CE}(\theta)$ can be evaluated by the overlap integral of the standing wave with the hole pattern $A(x, y)$ of the array [35], where $A(x, y)=1$ in a hole, and $A(x, y)=0$ outside the holes. A $\cos \theta$ term is also included to account for the projection of the FSP electric field, aligned along the propagation direction, onto the $x$ axis field transmitted by the array. The transmission is considered to be proportional to $\mathrm{CE}$, which is given by

$$
\mathrm{CE}(\theta)=\left|\int(\cos \theta) E_{\|}^{f s p} A(x, y) d S\right|,
$$

where the surface integral is over a section of the array. Here, we evaluate the overlap integral for a $990 \mu \mathrm{m} \times$ $990 \mu \mathrm{m}$ section of the array, consisting of 16.5 complete periods of the array in both the $x$ and $y$ directions. The relative amplitude of the calculated $\operatorname{CE}(\theta)$ shown as the solid line in Fig. 3 agrees well with the measured data.

The resonances have the following simple geometric interpretation: Starting along the $x$ axis for which $\theta=0$, the enhanced coupling angles $\theta_{n}$ are given by $\tan \theta_{n}=$ $n L /(M L)=n / M$, where $n=0,1, \ldots, 13$, and $M=16.5$ is the number of periods of the evaluated array along the $x$ axis. These angles are indicated on Fig. 3 by the vertical lines marked with the angle number. The agreement is quite good and gives support to our claim that we have
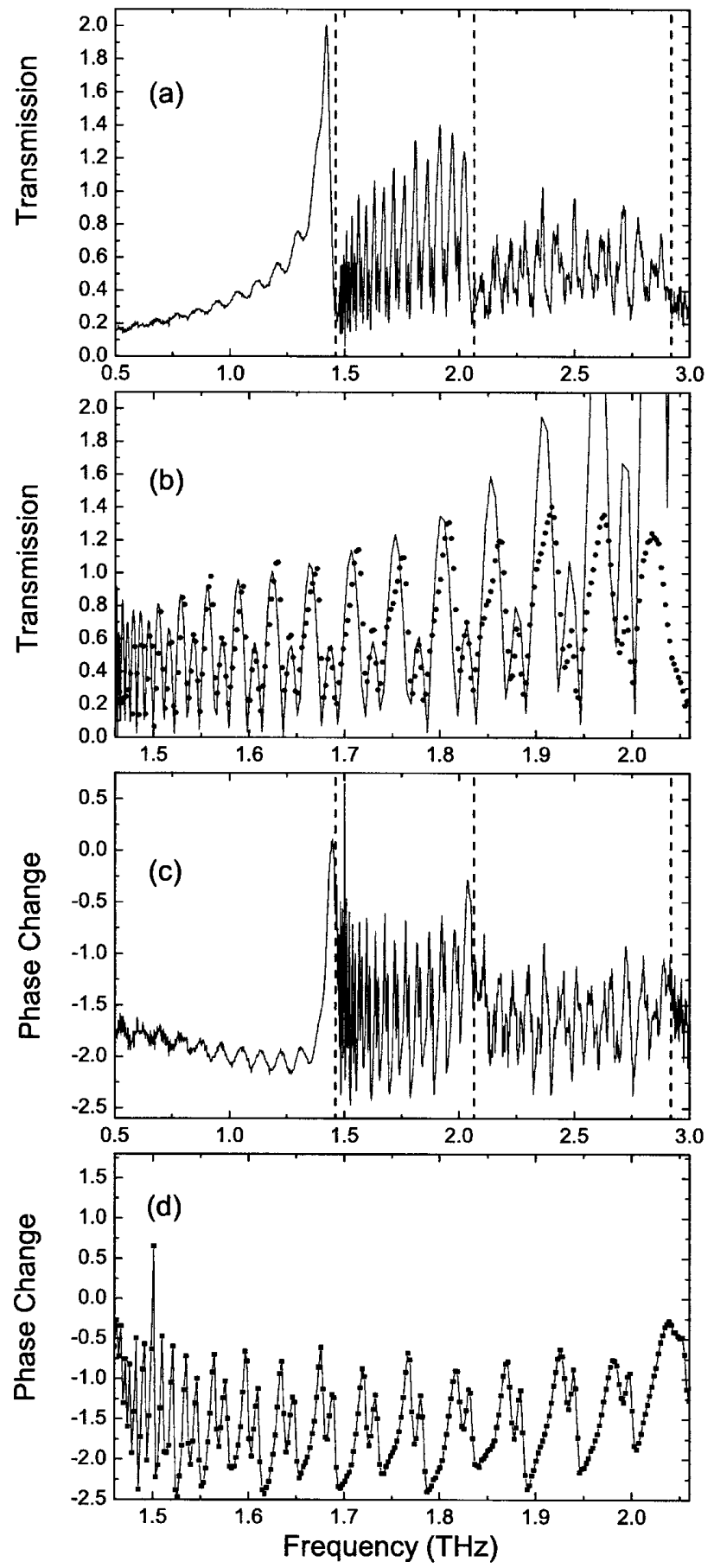

FIG. 2. (a) Experimental amplitude transmission. (b) Expanded experimental transmission (dots) compared with theory. (c) Experimental phase change in radians. (d) Expanded experimental phase change (dots), connected by a solid line to guide the eye. 


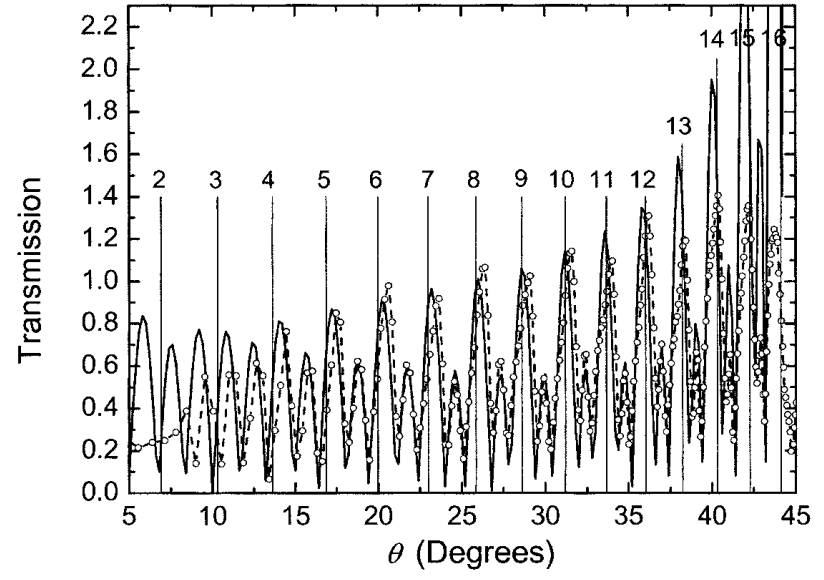

FIG. 3. Experimental amplitude transmission (open circles connected with a dashed line) compared with theory (solid line). The vertical lines mark $\theta_{n}$ from the simple geometric model.

excited surface plasmons propagating at these angles. Similarly, the smaller peaks at angles $\theta_{n+1 / 2}$ can be explained by the relationship $\tan \theta_{n+1 / 2}=(n+$ $1 / 2) L /(M L)$. These resonances are considered to be due to $L / 2$ length of the rectangular aperture.

The results are then transformed from $\theta$ to THz by the relationship $f=c / \lambda_{\operatorname{Res}}=c /\left(L n_{2} \cos \theta\right)$ and are presented in Fig. 2(b). The calculated CE, multiplied by an amplitude normalization factor, is shown as the solid line in Fig. 2(b), compared with the measured transmission. As shown in Fig. 2(b), our calculation matches the frequencies of the higher and lower measured FSP peaks and also the peaks' profile.

The array size used in the calculation provides the best match with experiment. Because of the binary treatment of the hole array in the overlap integral, we can consider (with no change in results) the size to be $1020 \mu \mathrm{m} \times 1020 \mu \mathrm{m}$, by slightly extending the original boundaries to the new corner locations (in microns) at the four-hole midpoints $(-22.5,-15)$, $(-22.5,1005),(997.5,-15),(997.5,1005)$. This new size with the same $17 \times 17$ hole locations consists of $17 \times 17$ complete array periods. Consider three additional, identical $1020 \mu \mathrm{m} \times 1020 \mu \mathrm{m}$ arrays symmetrically placed in contact, each with one corner at $(-22.5,-15)$. Because of the inversion symmetry of the resulting $2040 \mu \mathrm{m} \times 2040 \mu \mathrm{m}$, 4-array centered on $(-22.5,-15)$, the original overlap calculation also applies to the 4-array. The effective array size is considered to be determined by the spatial extent (at the array) of the planar phase coherence of the $\mathrm{THz}$ beam, due to the residual phase variation and curvature [36]. The $2 \mathrm{~mm} \times$ $2 \mathrm{~mm}, 4$-array is comparable in size to the spatial phase variations of an incoming $6 \mathrm{~mm}, 1 / e$ diameter. $1.5 \mathrm{THz}$ beam, and is thereby physically reasonable.
In summary, a new type of surface plasmons has been observed. By evaluating the overlap integrals of the corresponding standing waves with the hole pattern, we obtained good agreement with the experimental data, thereby confirming that the observed new resonances can be simply explained in terms of coupling between the standing waves and the $\mathrm{THz}$ waves transmitted through the hole pattern.

We thank Sharmila Rajendran for the hole array mask design and Abul Azad for help with the sample fabrication. This work was funded by the National Science Foundation and the Army Research Office.

[1] R. Ulrich, Infrared Phys. 7, 37 (1967).

[2] C.-C. Chen, IEEE Trans. Microwave Theory Tech. 18, 627 (1970); 21, 1 (1973).

[3] F. Keilman, Int. J. Infrared Millimeter Waves 2, 259 (1981).

[4] C. Winnewisser et al., Appl. Opt. 38, 3961 (1999).

[5] M. E. MacDonald et al., IEEE Trans. Microwave Theory Tech. 48, 712 (2000).

[6] C. Winnewisser et al., IEEE Trans. Microwave Theory Tech. 48, 744 (2000).

[7] T.W. Ebbesen et al., Nature (London) 391, 667 (1998).

[8] H. F. Ghaemi et al., Phys. Rev. B 58, 6779 (1998).

[9] T. J. Kim et al., Opt. Lett. 24, 256 (1999).

[10] T. Thio et al., J. Opt. Soc. Am. B 16, 1743 (1999).

[11] D. E. Grupp et al., Appl. Phys. Lett. 77, 1569 (2000).

[12] A. Krishnan et al., Opt. Commun. 200, 1 (2001).

[13] L. Martin-Moreno et al., Phys. Rev. Lett. 86, 1114 (2001).

[14] A. Dogariu et al., Opt. Lett. 26, 450 (2001).

[15] S. C. Hohgn et al., Appl. Phys. Lett. 81, 3239 (2002).

[16] A. Degiron et al., Appl. Phys. Lett. 81, 4327 (2002).

[17] E. Altewischer et al., Nature (London) 418, 304 (2002); J. Opt. Soc. Am. B 20, 1927 (2003); Opt. Lett. 28, 1906 (2003).

[18] E. Devaux et al., Appl. Phys. Lett. 83, 4936 (2003).

[19] D. S. Kim et al., Phys. Rev. Lett. 91, 143901 (2003).

[20] R. Gordon et al., Phys. Rev. Lett. 92, 037401 (2004).

[21] W. L. Barnes et al., Phys. Rev. Lett. 92, 107401 (2004).

[22] B. K. Minhas et al., J. Opt. Soc. Am. A 19, 1352 (2002).

[23] F. Miyamaru et al., Appl. Phys. Lett. 82, 2568 (2003).

[24] J. G. Rivas et al., Phys. Rev. B 68, 201306 (2003).

[25] A. Naweed et al., J. Opt. Soc. Am. B 30, 2534 (2003).

[26] M. Beruete et al., cond-mat 0311036.

[27] D. Qu et al., Opt. Lett. 29, 896 (2004).

[28] H. Cao and A. Nahata, Opt. Express 12, 1004 (2004).

[29] E. Popov et al., Phys. Rev. B 62, 16100 (2000).

[30] W. L. Barnes et al., Nature (London) 424, 824 (2003).

[31] S. A. Darmanyan and A.V. Zayats, Phys. Rev. B 67, 035424 (2003).

[32] R. Muller et al., Phys. Rev. B 68, 205415 (2003).

[33] A. M. Dykhne et al., Phys. Rev. B 67, 195402 (2003).

[34] D. Grischkowsky et al., J. Opt. Soc. Am. B 7, 2006 (1990).

[35] G. Gallot et al., J. Opt. Soc. Am. B 17, 851 (2000).

[36] M. T. Reiten et al., J. Opt. Soc. Am. B 20, 2215 (2003). 